\title{
Impact of universal screening on MRSA bacteremias in a single acute NHS organisation (2006-12): interrupted time-series analysis
}

\author{
Jayanta B Sarma ${ }^{*}$, Bryan Marshall ${ }^{\dagger}$, Victoria Cleeve ${ }^{\dagger}$, David Tate $^{\dagger}$ and Tamsin Oswald ${ }^{\dagger}$
}

\begin{abstract}
Background: In November 2004, a national target was set for the English hospital trusts to reduce the Meticillin-Resistant Staphylococcus aureus (MRSA) bacteremia rate by $60 \%$ by April 2008 against the number during 2003/04 (baseline year). In our organisation the number of MRSA bacteremias had risen since 2002 and peaked at 75 in 2005/06. A target was set to reduce the number and series of specific and non- specific interventions was introduced including universal MRSA screening. This study analyzes the impact of universal MRSA screening using a quasi-experimental design using routinely gathered data.
\end{abstract}

Methods: This study used data gathered routinely for clinical governance, quality control, financial management and outbreak monitoring purposes. Interrupted Time Series (ITS) analysis of 15 pre- and 19 post- universal MRSA screening (and decolonisation) quarterly numbers of bacteremias was carried out where Meticillin-Sensitive Staphylococcus aureus (MSSA) numbers served as non-equivalent dependent variable (control).

Results: An immediate sharp fall in MRSA bacteremias was observed following the universal MRSA screening (and decolonisation) commenced in Q2, 2007. The number dropped sharply from $23(\mathrm{Q} 2,2007)$ to $10(\mathrm{Q} 3,2007)$ for all MRSA bacteremias, and, from $15(\mathrm{Q} 2,2007)$ to $6(\mathrm{Q} 3,2007)$ for bacteremias $\geq 48$ hours of hospitalization. The declining trend continued reaching zero in Q2, 2009 and Q4, 2010 for those with $\geq 48$ hours of hospitalization and all bacteremias, respectively. ITS analysis revealed significant impact of universal MRSA screening on all MRSA bacteremias $\left(\beta_{2}-0.554, p 0.000\right)$ and those with $\geq 48$ of hospitalization $\left(\beta_{2}-0.577, p 0.001\right)$. Impact estimation predicted 17 and 13 bacteremias for all and those with $\geq 48$ hours hospitalization, respectively in the 19th quarter post-intervention, if the intervention did not occur. The number of MRSA isolates from non-blood culture systemic sources as percentage of admissions also dropped significantly from 3.32\% in Q2, 2007 to 1.51\% in Q3, 2007 $\left(\beta_{2}-0.506, p 0.000\right)$ which is still running low at $0.33 \%$ at the end of $Q 1,2012$. On the other hand, there was no statistically significant impact of universal screening on MSSA bacteremias.

Conclusions: We conclude that of all interventions, the universal MRSA screening (and decolonisation) is the most effective intervention associated with significant and sharp drop in MRSA burden.

\section{Background}

In November 2004, a national target was set for the English hospital trusts to reduce the Meticillin-Resistant Staphylococcus aureus (MRSA) bacteremia rate by $60 \%$ by April 2008 against the number during 2003/04 (baseline year). In our organisation the number of MRSA bacteremias had risen since 2002 and peaked at 75 in 2005/06. A target was set to reduce the number to 27 bacteremias in the year

\footnotetext{
* Correspondence: jayanta.sarma@nhs.net

${ }^{\dagger}$ Equal contributors

Northumbria Healthcare NHS Foundation Trust, North Shields NE29 8NH, UK
}

2007/08 and an improvement programme was initiated facilitated by Department of Health (DoH) in May 2006. A series of specific- and non-specific interventions was introduced since May 2006. The 'Clean your hands' campaign was already ongoing since September 2004. This study reviews the impact of these interventions to reduce the burden of MRSA bacteremias, specifically, the impact of universal MRSA screening using a quasi-experimental (Interrupted Time Series - ITS) design using routinely gathered data in the context of a comprehensive infection control program. 


\section{Methods}

The setting is an acute NHS Trust in the north east of England serving a mixed urban/rural predominantly elderly population of approximately 500,000. The Trust has three acute district general and seven community hospitals with 760 acute medical/elderly care beds, and 183 acute surgical beds including orthopedics. The average number of beds per ward is 21 with $82.9 \%$ bed occupancy. There is an active infection control team (ICT) with four point five whole time equivalent microbiologists, seven infection control nurses and input from other specialties including pharmacy. The study used no patient identifiable information and is a noninterventional observational study and as such did not require ethical approval.

\section{Data collection}

This study used data gathered routinely for clinical governance, quality control, financial management and outbreak monitoring purposes. The numbers of monthly bacteremias were routinely recorded for governance purposes. The review of surveillance (Sept 2004 to March 2006) data showed the sources of MRSA bacteremias to be unknown in an unusually large proportion (30\%) of cases, and in the rest, three major sources were identified viz., indwelling intra vascular devices, chest infections and soft tissue infections (Figure 1). Following the introduction of Root Cause Analysis (RCA) in May 2006 a number of interventions were made in quick succession as part of the MRSA improvement programme; implementation of these interventions and compliance levels were routinely monitored by audits (Table 1 ). Universal MRSA Screening and Decolonization was introduced in May, 2007. Before May, 2007 MRSA screening was selective based on certain risk factors including preoperative patients in elective surgery, emergency orthopedics and trauma surgery, critical care, patients known to be MRSA positive, oncology/chemotherapy inpatients and patients admitted from high-risk settings [1]. The hospitals screened only about 1600 patients per quarter before May, 2007. In May, 2007, the universal screening was introduced in a phased manner to include all adult elective, day case and emergency admissions. This was implemented in advance of the NHS Operating Framework for the NHS in England 2008/09 [2] which required screening for all elective admissions by April 2009 and all emergency admissions no later than 2011.

Swabs were placed on to MRSA Select ${ }^{\mathrm{TM}}$ (Bio-Rad) chromogenic agar medium and presumptive pink MRSA colonies were confirmed by a latex slide test. Full biochemical identification and antibiotic susceptibility were carried out by The VITEK ${ }^{\circledR} 2$ (bioMérieux UK) system. All positive MRSA results were communicated directly to the ICT. Average turnaround time was 48 hours.

The number of patients screened increased to 5000 in Q2, 2007 reaching 15000 in Q1, 2009 which has remained stable (Figure 2). A weekly MRSA screening audit commenced in September, 2007 showed near 100\% screening compliance achieved by beginning of April, 2009 for all elective and emergency admissions.

The MRSA positive patients were isolated according to the infection control policy and commenced on decolonisation treatment as soon they were identified irrespective of whether facilities were available to isolate. Octenisan $^{\circledR}$ (or $2 \%$ Triclosan) body wash once daily and Mupirocin 2\% Nasal ointment three times a day to the inside of each nostril for 5 days (and hair washing with Octenisan ${ }^{\circledR}$ on days 2 and 4 of the treatment) were

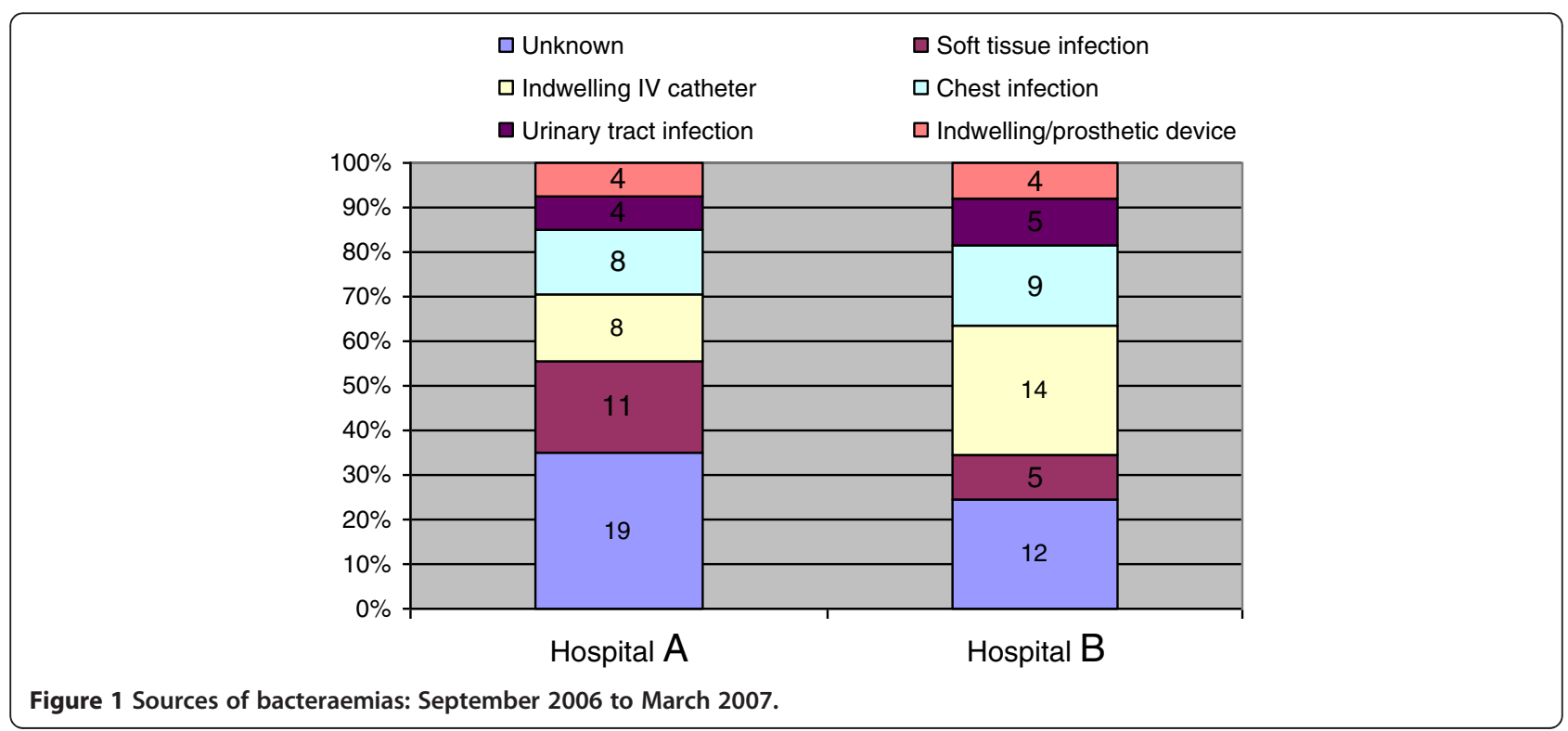


Table 1 Timeline of infection control interventions since Sep 2004

\section{Year Period Interventions}

2004 Sep 'Clean your hands' campaign [3]

2006 May Root cause Analysis (RCA)

\section{Description (compliance/comments)}

Compliance (against opportunities available) 62-72\% at baseline rising to $82 \%$ by April 2008. Intense daily monitoring continued with results aggregated and compiled weekly until 95\% compliance was achieved at which point frequency was reduced to weekly. In most locations within a few weeks the level of compliance rose to over $95 \%$.

The information capture tool was given to the clinical teams within hours of the identification of MRSA bacteremia. Once received back by the ICT further in depth investigation (if necessary) was carried out by the Infection Control Nurses (ICN). The clinical teams are updated during the regular visits by Microbiologists or ICNs. More formal feedback occurs during Clinical Governance or Operational board meetings, and also quarterly at Trust Board.

\begin{tabular}{|c|c|c|}
\hline Jul & Infection & Junior Doctor's Induction Video \\
\hline Sep & Control & Support Worker's Training \\
\hline Nov & Clinical & The peripheral vascular care (PVC) care \\
\hline
\end{tabular}

2007 May Universal Screening and decolonization

The central vascular care (CVC) care plan

Weekly HCAl meeting

Jul Improved blood culture technique ('Taking blood cultures: A summary of best practice' (DoH, June 2007)

\section{level management in terms of resources.}

Two one day audits in April 2007 and May 2007 showed improvement in usage. Another audit commenced on 22nd October 2007 for 37 consecutive weeks showed the number of cannula in situ for $>72$ hours was only a few and improved compliance to other elements of the care plan viz., (1) removal if no continued clinical indication, (2) use of care plan, (3) daily visual assessment and (4) intact dressing was observed within a few weeks and maintained throughout the period.

See result section for full description. 100\% compliance both for elective and emergency admissions achieved by beginning April 2009.

\section{The blood culture policy was re-issued which required that indication was} recorded in the medical notes and blood culture was authorised by a consultant/senior doctor. A training video was made available on the Trust intranet to demonstrate how to take cultures using aseptic technique. This was also shown at induction for new junior doctors. In June, the number of blood cultures taken fell from the monthly average of 1252 to 778 , a $38 \%$ reduction, of which $16 \%$ were positive compared to $14.5 \%$ in the previous five months. The proportion that was skin organisms (e.g., coagulase negative staphylococci, diptheroids and propionibacterium) marginally reduced to $24 \%$ from $29 \%$.

Patient Administration System (PAS) MRSA alert Patients with previous MRSA history were tagged with an alert code on the PAS to allow for decolonisation to commence within 24 hours of admission in accordance with the MRSA policy.

\section{Sep Standardised Intra Venous (IV) cannula site dressing}

Universal screening compliance audit See above May, 2007.

Annual infection control study day and road show

Oct PVC care plan compliance audit Web-based Audits Tool

\section{See above November, 2006.}

A web based data capture was introduced to audit MRSA screening, peripheral cannula care and hand hygiene standards. This new system enabled the ICT to produce weekly audit figures efficiently at review in the weekly HCAl group meeting, identify the outliers and giving real-time feed back to the ward staff to reinforce corrective measures and best practice in a targeted and timely way.

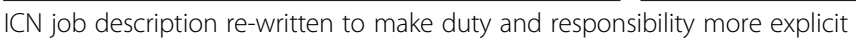


Table 1 Timeline of infection control interventions since Sep 2004 (Continued)

\begin{tabular}{|c|c|c|}
\hline & New infection control ward entrance sign & \\
\hline \multirow[t]{2}{*}{ Mar } & Management of screening positives & Daily meeting for management of screening positives \\
\hline & MRSA Screening at day 10 & $\begin{array}{l}\text { Screening of all inpatients at day ten after admission (and then every ten } \\
\text { days). }\end{array}$ \\
\hline \multirow[t]{2}{*}{ Apr } & Hand hygiene audit & See above Sep 2004 ('Clean your hands' campaign) \\
\hline & Aseptic training for the staff & \\
\hline \multirow[t]{2}{*}{ June } & Junior doctor's e-learning on Infection Control & \\
\hline & Fluoroquinolone restriction & $\begin{array}{l}\text { Consumption dropped from average } 12 \text { Defined daily Dose (DDD)/100 bed- } \\
\text { days in previous } 12 \text { months to under } 5 \text { from June } 2008 \text { with a further drop } \\
\text { in consumption to under } 2 \text { from June } 2010 \text {. }\end{array}$ \\
\hline
\end{tabular}

used for decolonisation. A Patient Group Directive (PGD) for prompt prescribing of Octenisan ${ }^{\circledR}$ (or $2 \%$ Triclosan) washes by the nursing staff was already in place. Patients are re-screened 48 hours after completing decolonisation if they are still in hospital. In general, up to two complete courses of decolonisation were given during each inpatient stay. Any patient who was still positive for MRSA after a second decolonisation treatment received daily washes using octenisan for the duration of their in-patient stay.

\section{Analysis}

For the interrupted time-series (ITS) analysis, we used segmented linear regression, which divides a time series into pre- and post-intervention segments. The universal screening was introduced in Q2, 2007, therefore, Q3, 2007 was chosen as the intersection between the preand post-intervention segments. Interventions that may be short-lived may erroneously report maximal effects if short time series are analyzed; our time series included 19 pre- and 15 post-intervention quarterly data points.
A linear regression model has two parameters: the level and slope. Therefore the difference between the two segments can be quantified by testing the change in these two parameters (Equation 1). A change in level between the pre- and post-intervention segments indicates a step-change, and a change in slope indicates a change in trend.

$$
Y_{t}=\beta_{0}+\beta_{1} T+\beta_{2} D+\beta_{3} P
$$

$\mathrm{Y}_{\mathrm{t}}$

is the MRSA rate at month $\mathrm{t}$;

$\beta_{0}$

$\beta_{1}$

estimates the baseline MRSA quarterly rate;

estimates the baseline (pre-intervention) linear trend where $\mathrm{T}$ is a continuous variable indicating the time in quarter interval at time $t$ from the start of the study period;

$\beta_{2}$ estimates the level change between pre- and post-

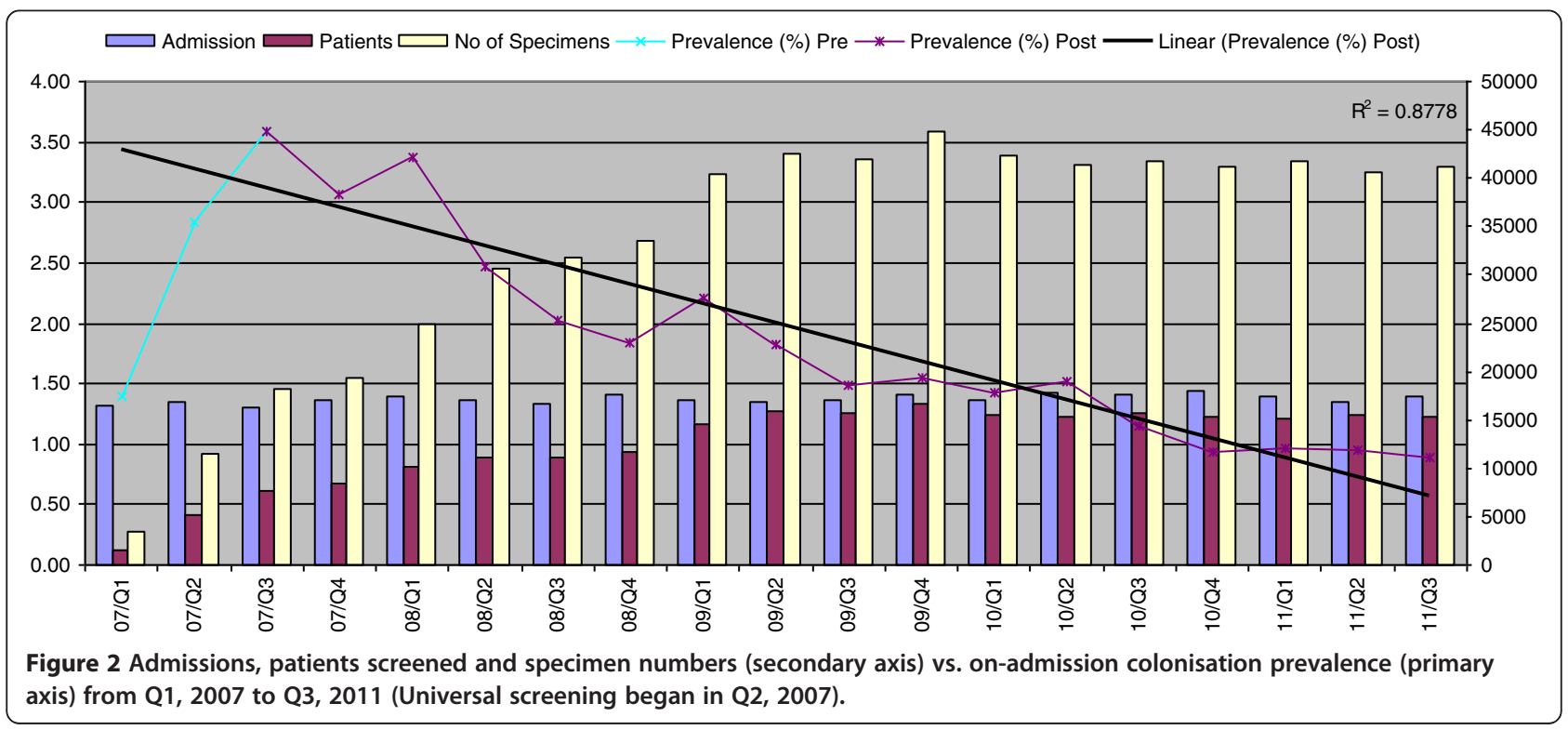


intervention, where $\mathrm{D}=0$ before the intervention, and $\mathrm{D}=1$ after the intervention;

$\beta_{3}$

estimates the mean quarterly trend in MRSA postintervention, compared to the baseline trend, where $\mathrm{P}$ is a continuous variable indicating the number of quarters after the start of the intervention at time $t$ and is coded as zero before the intervention.

Three points of interest were calculated: (1) preintervention trend (slope); (2) post-intervention change of the baseline level representing an immediate effect; and (3) post-intervention trend (slope) representing a sustained effect of the intervention. A $p$-value of 0.05 was regarded as statistically significant. If coefficient $\beta_{1}$ was significantly different from 0 , the pre-intervention trend was deemed statistically significant. Similarly, if $\beta_{2}$ and/or $\beta_{3}$ were significantly different from 0 , it was assumed that there had been a significant postintervention change of baseline level and/or trend.

We included Meticillin-Sensitive Staphylococcus aureus (MSSA) numbers for the same period as nonequivalent dependent variable [5] which would be affected by all infection control interventions except the MRSA universal screening. ITS analysis for both MRSA and MSSA series was carried out, Q3, 2007 as the intersection between segments. The null hypothesis was that changes in the positive blood cultures and positive systemic samples other than blood cultures for MRSA and the MSSA were similar.

We have included Durbin-Watson (DW) in the regressions statistics to correct for possible auto-correlation. DW value between 1.5 and 2.5 indicates that the values are independent. All analysis was done using SPSS 19.

\section{Results \\ Patients screened}

The number of patients screened almost trebled from 1642 (3,432 specimens) in Q1, 2007 to 5087 (11,518 specimens) in Q2, 2007 then increased gradually over the next quarters to finally stabilize over 15000 (40,000 specimens) by Q1, 2009 (Figure 2). The ratio of patients to specimen numbers was about 1:2 in line with the swabbing of nose $(\mathrm{N})$ and throat $(\mathrm{T})$ only. However, this ratio has increased since Q2, 2008 to just under 1:3 when we introduced inpatient MRSA screening at every 10 days interval. The highest detection of on-admission prevalence of colonized patients was achieved (3.59\%) in the Q3, 2007 then sharply reduced to $2 \%, \mathrm{Q} 2,2008), 1 \%, \mathrm{Q} 3,2010)$ and, finally, dipped down to $0.89 \%$ in $Q 4,2010\left(R^{2}=0.9\right)$, clearly reflecting the efficacy of decolonization and successful eradication (Figure 2).

\section{Estimation of baseline prevalence of MRSA colonization}

The highest rate of MRSA positive systemic samples (infections) other than blood cultures was $6.47 \%$ of all admissions in Q1, 2005 which implies a rate of colonization prevalence much higher than $6.47 \%$. What was the on-admission colonization rate could not be extrapolated from inpatient infection burden but in Q1, 2007 the on-admission colonization detection rate was only $1 \%$ with risk based targeted screening. The highest on-admission detection rate of $3.59 \%$ was achieved in Q3, 2007 after universal screening was commenced (Figure 2). The impact of the universal screening and decolonisation on the MRSA burden was immediate: there was a sharp decline in on-admission colonisation prevalence from a peak at $3.59 \%$ in Q3, 2007 to less than $1 \%$ by Q4, 2010, while the number of patients screened remained stable at just over 15,000/quarter. These data reflect that detection by targeted screening before the universal screening was by far less efficient.

\section{Impact on MRSA bacteremias}

The target set required a reduction from 67 to 27 bacteremias by April, 2008 equivalent to numbers falling from 5.6 cases to 2.3 cases per month compared to the baseline year. Initially, following the introduction of RCA in May 2006, a tangible reduction in the numbers was achieved on a month by month basis. A care pathway for insertion, management and maintenance of central and peripheral lines was introduced (November, 2006). However, in the subsequent months there was a gradual increase with MRSA bacteremias peaking at 23 cases in Q2, 2007. The universal MRSA screening was introduced in Q2, 2007 and an immediate and drastic reduction in MRSA bacteremias was seen in Q3, 2007; the declining trend continued till Q1, 2012 with only two cases $\geq 48$ h of hospitalization (hospital acquired) occurring in 2011-12.

Visual inspection of data (Excel graphics) revealed an immediate effect of universal screening in terms of sharp decline of MRSA bacteremias as well as on positive systemic (non-blood culture) samples in line with the decline of MRSA on-admission prevalence.

There was an immediate sharp fall in all MRSA bacteremias from 23, Q2 2007 to 10, Q3, 2007 followed by a continued declining trend reaching zero Q4, 2010 (Figure 3). Predicted regression line showed a slight decreasing trend in the slope before the intervention, an abrupt drop in the rate immediately following the intervention, and a gradually decreasing slope continued after the intervention. The hospital acquired MRSA bacteremias ( $\geq 48$ hours of hospitalization) dropped from 15 in Q2 2007 to 6 in Q3, 2007 followed by a continued declining trend reaching zero in Q2, 2009 (Figure 4). These 


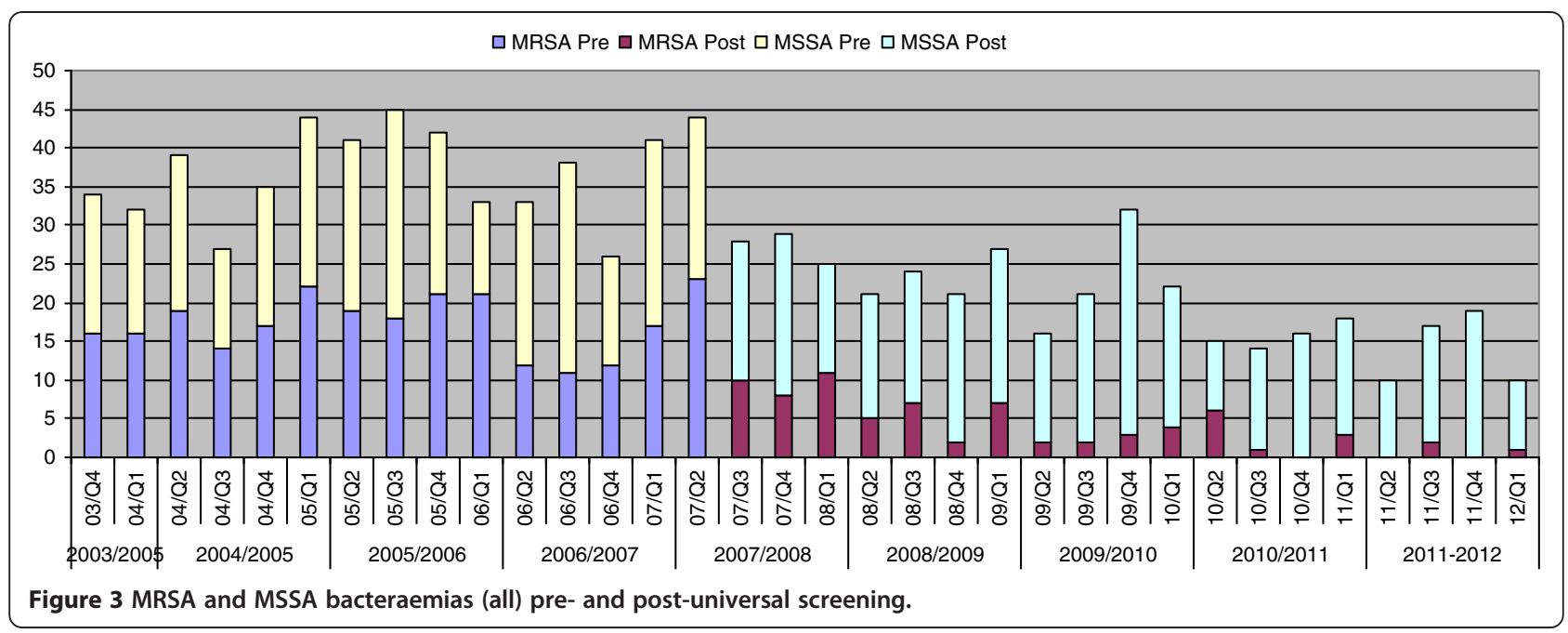

observations were tested using interrupted time-series (ITS) analysis. The ITS analysis (Table 2) revealed significant post-intervention drop of the baseline level of all MRSA bacteremias, representing an immediate effect, $\left(\beta_{2}-0.554, p 0.000\right)$ and the post-intervention declining trend (slope), representing a sustained effect $\left(\beta_{3}-0.393\right.$, $p$ 0.048) where pre-intervention coefficient $\beta_{1}$ was close to $0(-0.033$,$) signifying a flat trend. The immediate effect was$ equally prominent for hospital acquired MRSA bacteremias i.e., $\geq 48 \mathrm{~h}$ of hospitalization $\left(\beta_{2}-0.577, p 0.001\right)$ but post-intervention slope was not statistically significant $\left(\beta_{2}-0.216, p 0.298\right)$ as the numbers were already very low after the immediate sharp drop.

Impact estimation predicted 17 (all) and 13 ( $\geq 48$ hours of hospitalization) MRSA bacteremias, respectively in the 19th quarter post-intervention if the intervention did not occur, and, predicted zero bacteremias with intervention which is borne out by the observation.
There was no statistically significant impact of universal screening on MSSA bacteremias. During the whole observation period of 34 consecutive quarters the number of MSSA bacteremias, both community onset $\left(R^{2}=0.13\right)$ and $\geq 48$ hours of hospitalization $\left(R^{2}=0.09\right)$ have been declining slowly but not at a statistically significant level (Figure 5, Table 2).

\section{Impact on systemic Non-blood culture specimens}

Similarly, there has been an equally significant impact on the number of MRSA isolates from non-blood culture systemic sources: sterile fluid, tissues, respiratory specimens and swabs, superficial and deep (Figure 6). The number of positive specimens as percentage of admissions dropped from $3.32 \%$ in Q2, 2007 to $1.51 \%$ in Q3, 2007. The declining trend continued steadily over the following quarters to the lowest $0.25 \%$ in Q4, 2010 $\left(R^{2}=8.9\right)$. The ITS analysis revealed a pre-intervention

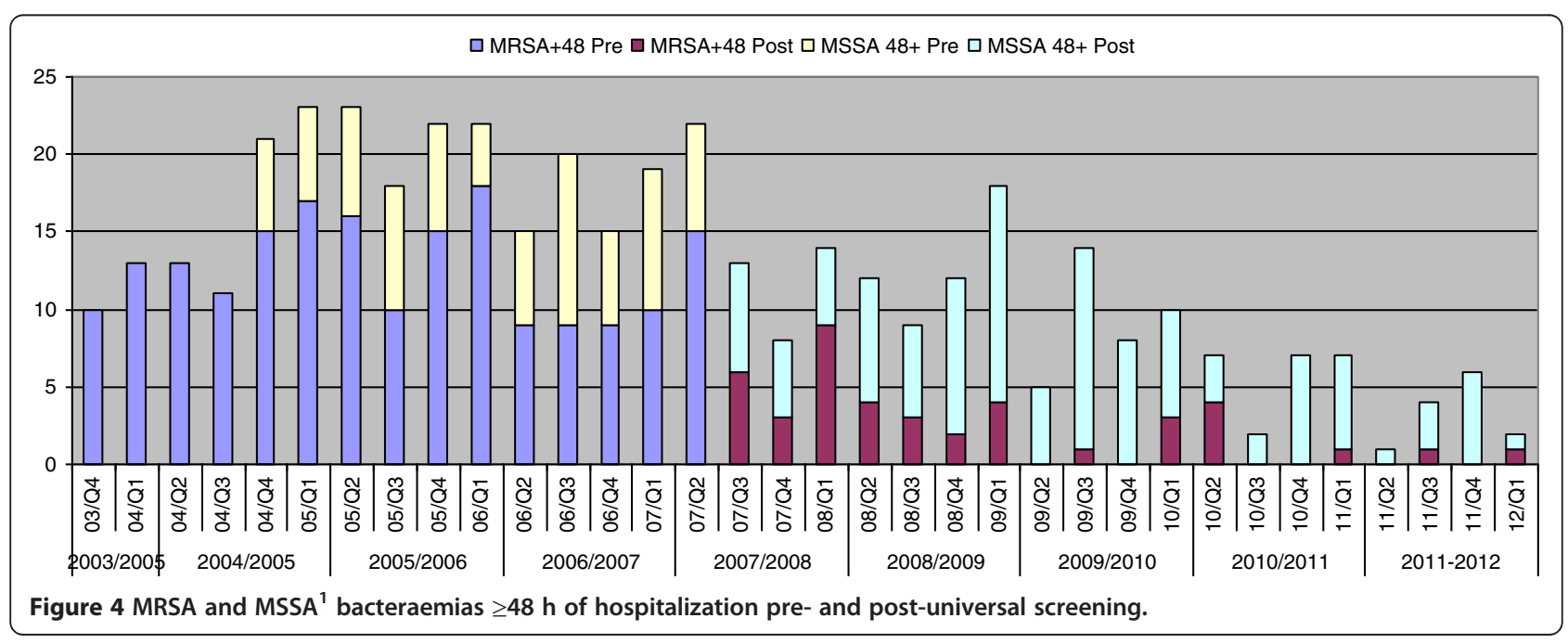


Table 2 Interrupted time-series regression analysis of the MRSA/MSSA bacteremias (total and $\geq 48$ h), MRSA/MSSA systemic (Hospital - \% of admissions) and MRSA/MSSA systemic (Community - total numbers)

\begin{tabular}{|c|c|c|c|c|c|c|c|}
\hline & Parameter & $\begin{array}{l}\text { Unstandardized } \\
\text { Coefficients - B (Std. Error) }\end{array}$ & $\begin{array}{l}\text { Standardized } \\
\text { Coefficients - } \beta\end{array}$ & t & $p$ & $95 \% \mathrm{Cl}$ & D-W \\
\hline \multirow[t]{4}{*}{ MRSA bacteremias } & Constant & $17.40(1.64)$ & & 10.59 & .000 & 14.04 to 20.75 & 1.573 \\
\hline & Pre-intervention trend & $-.025(.181)$ & -.033 & -.138 & .891 & -.39 to .34 & \\
\hline & Post-intervention change & $-8.32(2.072)$ & -.554 & -4.016 & .000 & -12.55 to -4.09 & \\
\hline & Post-intervention trend & $-.45(.221)$ & -.393 & -2.065 & .048 & -.90 to -.005 & \\
\hline \multirow[t]{4}{*}{ MSSA bacteremias } & Constant & $17.36(2.45)$ & & 7.075 & .000 & 12.35 to 22.37 & 2.068 \\
\hline & Pre-intervention trend & $.29(.270)$ & .604 & 1.098 & .281 & -.255 to .848 & \\
\hline & Post-intervention change & $-2.28(3.095)$ & -.235 & -.737 & .467 & -8.60 to 4.04 & \\
\hline & Post-intervention trend & $-.61(.330)$ & -.818 & -1.858 & .073 & -1.28 to. 06 & \\
\hline \multirow[t]{4}{*}{ MRSA $\geq \mathbf{4 8} \mathrm{h}$ bacteremias } & Constant & $13.46(1.38)$ & & 9.75 & .000 & 10.64 to 16.28 & 1.753 \\
\hline & Pre-intervention trend & $-.10(.152)$ & -.168 & -.659 & .515 & -.41 to .21 & \\
\hline & Post-intervention change & $-6.79(1.741)$ & -.577 & -3.901 & .001 & -10.34 to -3.23 & \\
\hline & Post-intervention trend & $-.19(.185)$ & -.216 & -1.060 & .298 & -.57 to .18 & \\
\hline \multirow[t]{4}{*}{ MSSA $\geq \mathbf{4 8} \mathrm{h}$ bacteremias } & Constant & $5.96(1.84)$ & & 3.22 & .003 & 2.16 to 9.76 & 1.901 \\
\hline & Pre-intervention trend & $.173(.272)$ & .503 & .634 & .532 & -.38 to .73 & \\
\hline & Post-intervention change & $1.10(2.112)$ & .178 & .521 & .606 & -3.24 to 5.44 & \\
\hline & Post-intervention trend & $-.45(.298)$ & -.990 & -1.524 & .140 & -1.06 to .15 & \\
\hline \multirow[t]{4}{*}{ MRSA Systemic (Trust) } & Constant & $5.8(.225)$ & & 25.78 & .000 & 5.34 to 6.26 & 1.599 \\
\hline & Pre-intervention trend & $-.17(.028)$ & -.830 & -6.252 & .000 & -.23 to -.11 & \\
\hline & Post-intervention change & $-2.03(.271)$ & -.506 & -7.493 & .000 & -2.58 to -1.47 & \\
\hline & Post-intervention trend & $.10(.033)$ & .350 & 3.269 & .003 & .040 to 17 & \\
\hline \multirow[t]{4}{*}{ MSSA Systemic (Trust) } & Constant & $3.25(.191)$ & & 17.01 & .000 & 2.86 to 3.64 & 1.421 \\
\hline & Pre-intervention trend & $-.05(.024)$ & -.892 & -2.358 & .026 & -.10 to -.007 & \\
\hline & Post-intervention change & $.29(.230)$ & .248 & 1.289 & .208 & -.17 to .76 & \\
\hline & Post-intervention trend & $.134(.028)$ & 1.477 & 4.846 & .000 & .07 to .19 & \\
\hline \multirow[t]{4}{*}{ MRSA Systemic (GP) } & Constant & $287(12.35)$ & & 23.25 & .000 & 262 to 313 & 1.962 \\
\hline & Pre-intervention trend & $-2.49(1.55)$ & -.285 & -1.602 & .120 & -5.68 to .69 & \\
\hline & Post-intervention change & $-66.23(14.89)$ & -.402 & -4.447 & .000 & -96.73 to -35.72 & \\
\hline & Post-intervention trend & $-4.22(1.78)$ & -.338 & -2.362 & .025 & -7.88 to -.56 & \\
\hline \multirow[t]{4}{*}{ MSSA Systemic (GP) } & Constant & $906(31.57)$ & & 28.71 & .000 & 841 to 971 & 2.427 \\
\hline & Pre-intervention trend & $-7.52(3.97)$ & -1.27 & -1.891 & .069 & -15.67 to .62 & \\
\hline & Post-intervention change & $11.71(38.04)$ & .105 & .308 & .760 & -66.22 to 89.65 & \\
\hline & Post-intervention trend & $10.16(4.56)$ & 1.20 & 2.224 & .034 & .80 to 19.51 & \\
\hline
\end{tabular}

declining trend $\left(\beta_{1}-0.830, p .000\right)$ but a significant sharp post-intervention drop representing an immediate effect $\left(\beta_{2}-0.506, p 0.000\right)$. However, there was no prominent post-intervention declining trend $\left(\beta_{3} 0.350\right.$, $p$ 0.003) as the rate already dropped and was running at a very low level at $<0.3 \%$. Percentage of MRSA of all S. aureus isolates (non-blood culture systemic sources) declined from 51\% Q2, 2007 (peak was at $67.54 \%$ in Q3, 2005) through 34\% Q3, 2007 to $8.7 \%$ in Q1, 2012.

The immediate decline and post-intervention declining trend were also significant for MRSA non-blood culture systemic specimens from general practitioners
(GP): $\beta_{2}-0.402, p 0.000 ; \beta_{3}-0.338, p 0.025$ whereas pre-intervention declining trend was not significant (Table 2).

Of note, however, there was no concomitant reduction in MSSA isolates from non-blood culture systemic sources observed. Instead, there was a slow but statistically significant post-intervention increasing trend in the rate of hospital MSSA $\left(\mathrm{R}^{2}=7.3\right.$, Figure 7$)$ confirmed by ITS analysis $\left(\beta_{3}\right.$ 1.477, $p$ 0.000). The ITS analysis also revealed post-intervention increasing trend in the numbers of non-blood culture systemic MSSA from GP $\left(\beta_{3}\right.$ 1.20, $p$ 0.034) although the predicted regression line was not significant $\left(R^{2}=0.06\right)$. 


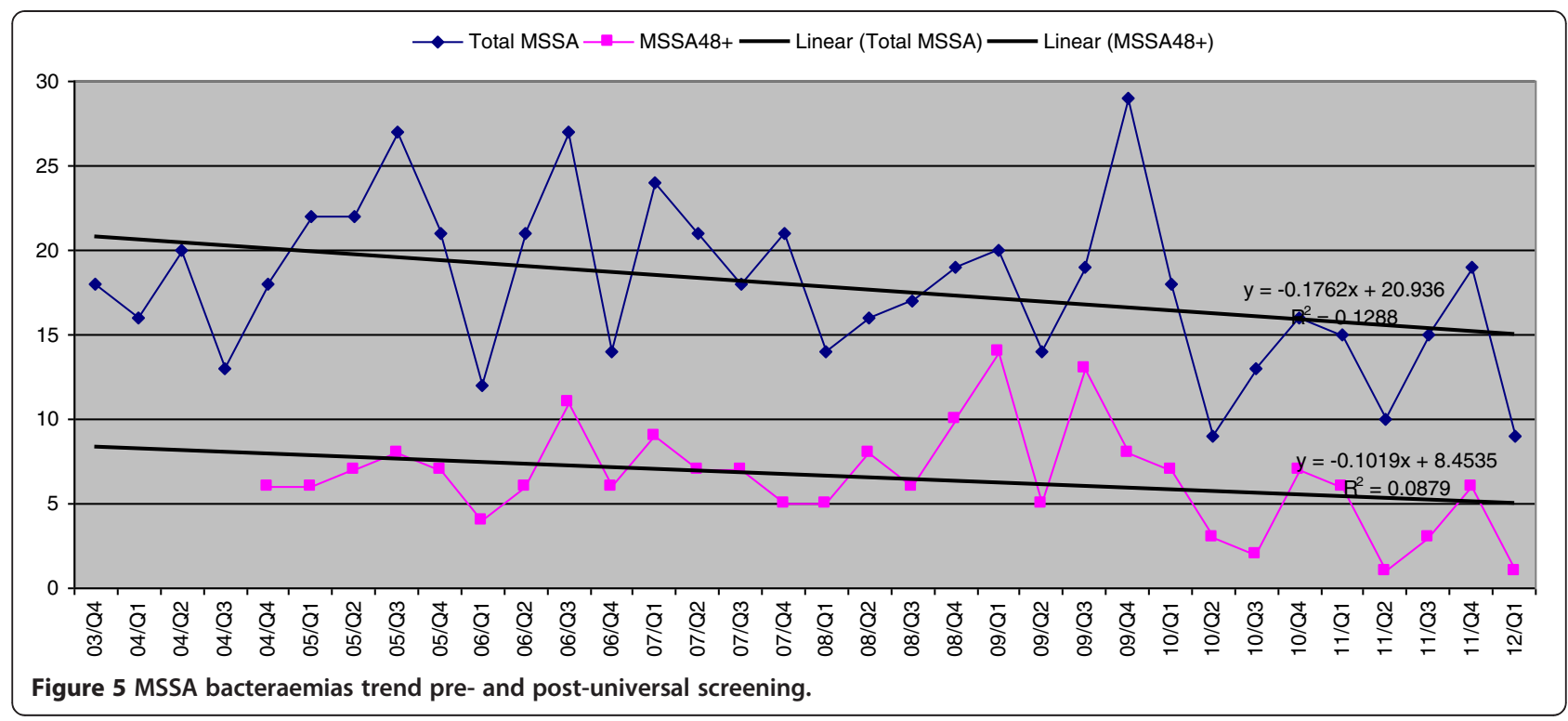

\section{Cost of screening}

Each specimen was associated with a consumable cost of $£ 0.62$ for a negative screen and, additional, £2.16 for a positive specimen (identification and sensitivity). Estimated cost of screening was $£ 24,800$ per quarter plus $£ 1080$ based on the current quarterly positive number of about 500. The yearly cost of universal screening was just over $£ 100,000$ for laboratory consumables alone. In Q1, 2012 a combined NP Elution Swab (ESwab) in Liquid Amies medium was introduced reducing the number of specimens to half. The Eswab costs $£ 0.45$ more compared to the standard Amies swab, so, total consumable cost would be only marginally lower but specimen numbers halved.

\section{Mupirocin resistance}

The resistance to mupirocin (hospital isolates) marginally increased from $1.7 \%$ in 2004 to $2.3 \%$ in 2011.

\section{Conclusion}

In the era of multi-resistant pathogens, focusing hospital resources on a single antibiotic-resistant pathogen as a sole approach to infection control is criticized as inherently flawed [6]. Questions were also raised regarding the impact of universal screening on MRSA bacteremia rate given the sensitivity of the screening, lack of isolation rooms, the relatively low effectiveness of current decolonization techniques, staffing time and financial resources required vis-à-vis (cost-) effectiveness and the

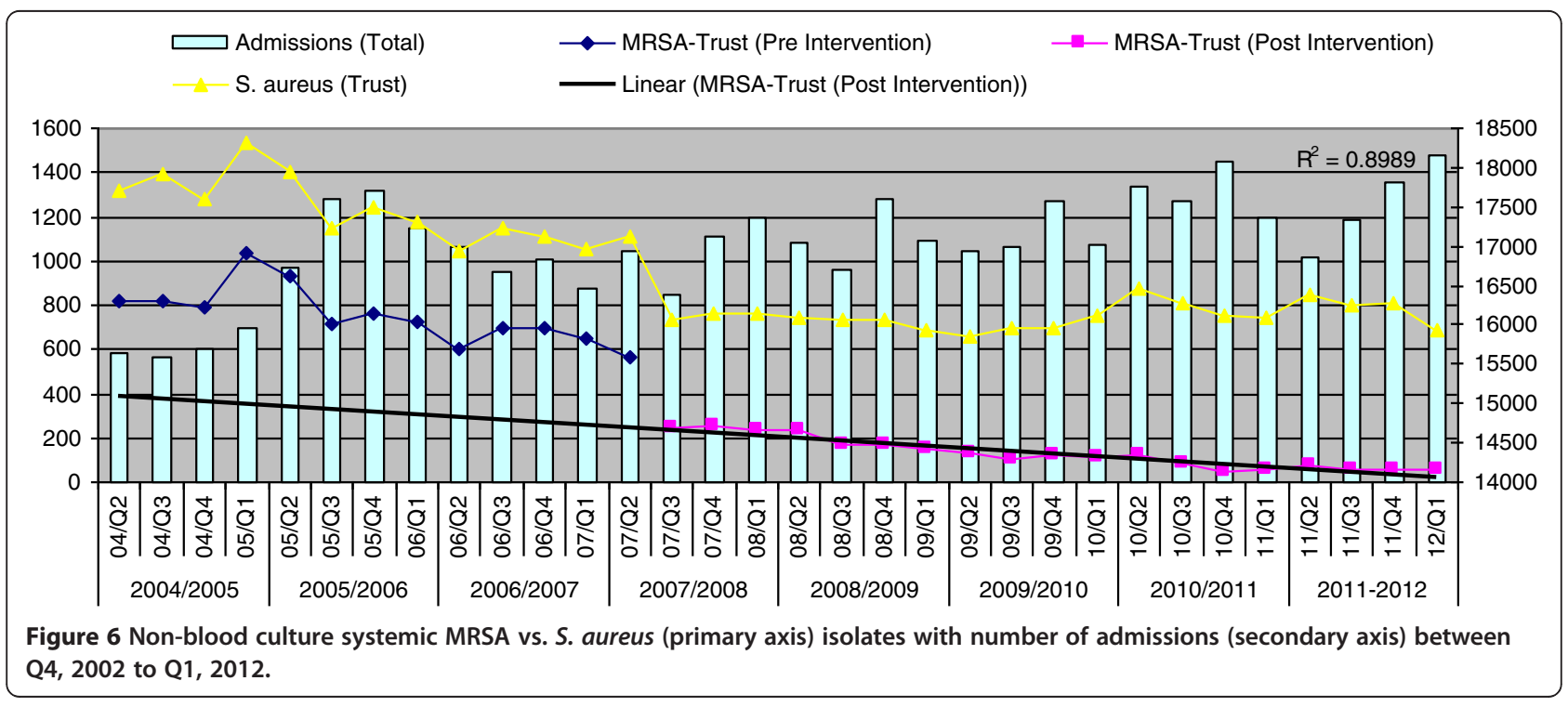




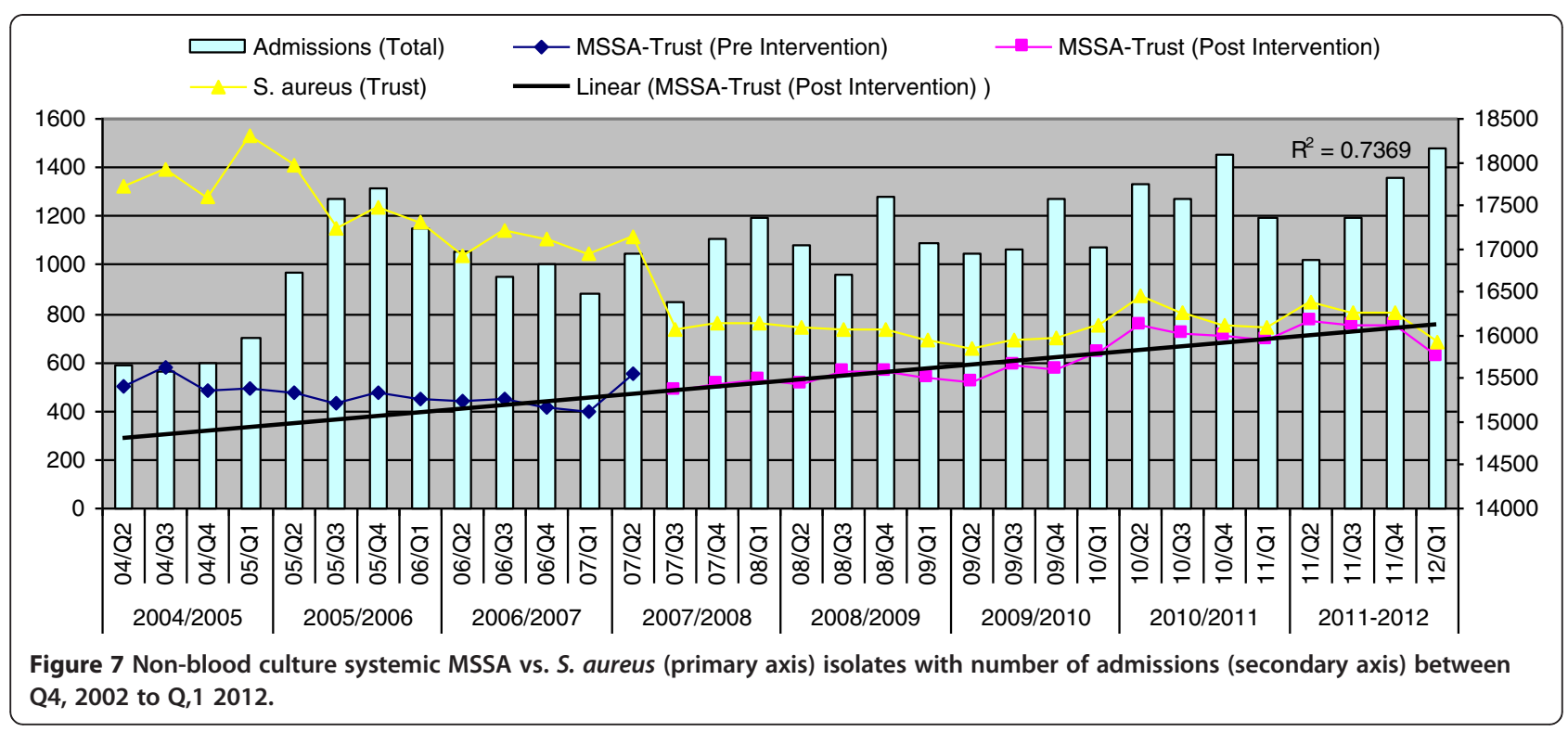

real reduction in risk achieved. Caution is advised for more evidence before it becomes routine considering resource requirements, practical difficulties and consequences [7]. In hospitals with an MRSA on-admission prevalence of $<5 \%$ targeted rather than universal screening is advocated [8].

In our study, all interventions were targeted at both MRSA and MSSA except the screening and decolonization but decline in MSSA bacteremias over the 34 consecutive quarters of observation was not statistically significant, which served as a natural control (non-equivalent dependent variable) in the analysis. In contrast, a drastic drop and quarterly declining trend in MRSA bacteremias continued, reaching zero following the introduction of universal screening. In national context, during the first 2 years following setting of the national target in November 2004, there was minimal reduction; however, from September 2006 onwards, rates declined dramatically to reach a reported 57\% reduction by April-June 2008 [9-11]. In our organization, dramatic decline in MRSA bacteremias and systemic infections was clearly linked to the introduction of universal screening and decolonization. This is consistent with reports that widespread uptake of decolonization has made the key additional contribution [11,12]. The effect of hand hygiene, identification of patients with MRSA infections or colonization and isolation interventions alone leads only to a gradual reduction in MRSA burden over many years [13]. The Pathfinder project [14], an implementation project to evaluate the impact of universal screening recommended by the NHS QIS HTA modeling [15], also found a temporal association between the initiation of universal screening and a significant reduction in colonization from $5.5 \%$ to $3.5 \%$ by month 12 . However, no significant reduction in the rate of MRSA bacteremias and Surgical Site Infections (SSI) during the study was found. A re-worked model using observed parameters from the Pathfinder study projected that colonization prevalence could reduce over three to five years to low endemic levels (0.5-1.8\%) with a chromogenic agar based universal screening strategy. Our study results support this projection.

In one neighbouring NHS Trust only seven additional day case patients in one month period would have been identified as MRSA carriers using the DoH universal screening compared with their targeted approach [16]. However, this Trust had already embarked on an expanded screening programme in three phases and needed to extend the screening only to day cases and elective admissions to comply with mandatory universal screening commenced in April, 2009. It is only to be expected that a successful screening and decolonization programme over a period would eventually lower the burden and a corresponding fall in MRSA detection rate.

In previous studies fluoroquinolone restriction was associated with decline in MRSA infections [17]. In our study MRSA rates dropped during the period when fluoroquinolone were used unrestricted albeit at a low level of average 12 DDD/100 bed-days per month. Our Fluroquinolone consumption more than halved to under 5 DDD/bed-days from June, 2008 which further dropped to under 2 from June, 2010. This low level of consumption may have positively impacted on our sustained low burden of MRSA infections.

Once low on-admission prevalence has been consolidated and sustained, a first line 'risk assessment' screening tool to reduce swabbing, laboratory costs and 
identify the proportion of high risk patients who could be preemptively isolated is a logical way forward. One study found that a simple three question Clinical Risk Assessment (CRA) could perform to a similar level to universal single site swabbing, but with considerably reduced resource implications [15]. The CRA has to be based on the local MRSA epidemiology, infection control practices and vulnerability of the patient population.

The ITS methods used in this study have several advantages over other quasi-experimental studies, because they are less likely to be influenced by certain biases [18-20]. Cyclical effects and underlying increasing or decreasing secular trends may contribute to observed intervention effects. While looking at the effect of specific intervention, the analysis has to take into account the added effect of pre-existing or newly introduced measures. Finally, auto-correlated data means that adjacent data points can be more similar (positive auto-correlation) or dissimilar (negative autocorrelation), leading to under- or over-estimates of effect, respectively. Unlike the classical statistical methods that assume the observed data are independent random variables, time-series analysis takes into account the relationships existing between consecutive observations, a phenomenon known as auto-correlation. However, as with all non-experimental designs, causal inference from ITS designs is limited because it is impossible to rule out alternative explanations for observed changes in the time series.

Our experience with the precipitous decline in the burden of MRSA provides clear evidence of the efficacy of universal screening and decolonization. We achieved a very high level of compliance with screening uptake and decolonization. However, this success has to be considered in the context of the highly empowered weekly HCAI meeting in operation since May, 2007. This group have been ensuring continued compliance to MRSA screening, PVC care plan use and hand hygiene reviewing weekly audits and feeding back to the relevant staff with timely recommendation of appropriate measures backed by the support from the management.

It is noteworthy that our success was in the background of a declining trend in MRSA infections in England [11], the USA [21] and elsewhere during the period. If there is an epidemiological explanation, perhaps, an effect predicated on natural biological trends involving specific MRSA strains is a matter of great interest $[11,22,23]$.

The study reveals one disturbing trend. The rate of non-blood culture systemic MSSA positive samples from the trust since Q2, 2007 is increasing. Also, the MSSA bacteremia trend appears to be decreasing only slowly and not at a significant level. This warrants specific actions to combat the MSSA burden.

\section{Competing interests}

The authors declare that they have no competing interests.

\section{Authors' contributions}

JBS prepared the manuscript. All authors contributed to the analysis and reviewed the manuscript.

\section{Authors' information}

BM is Director of Infection Control and Prevention.

JBS, VC, DT and TO are Consultant Microbiologists and Infection Control Doctors.

\section{Acknowledgment}

We acknowledge the contribution of the every single member of the ICT and strong management support which led to the success in controlling the MRSA in our organisation.

The publication of this article has been made possible by an unrestricted educational grant from Novartis Pharmaceuticals UK Limited.

Received: 13 September 2012 Accepted: 6 January 2013

Published: 14 January 2013

\section{References}

1. Screening for Meticillin-resistant Staphylococcus aureus (MRSA) colonisation: a strategy for NHS trusts: a summary of best practice: 2006. http://www.dh. gov.uk/prod_consum_dh/groups/dh_digitalassets/@dh/@en/documents/ digitalasset/dh_063187.pdf.

2. NHS Operating Framework for the NHS in England: 2008. http://www.dh gov.uk/en/Publicationsandstatistics/Publications/ PublicationsPolicyAndGuidance/DH_081094.

3. Patient safety alert 04: National patient safety agency. 2004. http://www.npsa. nhs.uk/EasySiteWeb/GatewayLink.aspx?alld = 75060 .

4. Reducing healthcare associated infections: High impact interventions: department of health. 2010. http://webarchive.nationalarchives.gov.uk/ 20120118164404/http://hcai.dh.gov.uk/whatdoido/high-impactinterventions.

5. Shadish WR, Cook TD, Campbell DT: Experimental and quasi-experimental designs for generalized causal inference. Belmont, Calif: Wadsworth Cengage Learning; 2002.

6. Wenzel RP, Bearman G, Edmond MB: Screening for MRSA: a flawed hospital infection control intervention. Infect Contr Hosp Epidemiol 2008, 29:1012-1018

7. Dancer SJ: Considering the introduction of universal MRSA screening. J Hosp Infect 2008, 69(4):315-320.

8. Harbarth S, Hawkey PM, Tenover F, Stefani S, Pantosti A, Struelens MJ: Update on screening and clinical diagnosis of meticillin-resistant staphylococcus aureus (MRSA). Int J Antimicrob Agents 2011, 37:110-117.

9. Pearson A, Chronias A, Murray M: Voluntary and mandatory surveillance for methicillin-resistant Staphylococcus aureus (MRSA) and methicillinsusceptible S. aureus (MSSA) bacteraemia in England. J Antimicrob Chemother 2009, 64(Suppl 1):11-17. doi:10.1093/jac/dkp260.

10. Ellington MJ, Hope R, Livermore DM, Kearns AM, Henderson K, Cookson BD, Pearson A, Johnson AP: Decline of EMRSA-16 amongst methicillinresistant Staphylococcus aureus causing bacteraemias in the UK between 2001 and 2007. J Antimicrob Chemother 2010, 65:446-468. doi:10.1093/jac/dkp448.

11. Wilson J, Elgohari S, Livermore DM, Cookson B, Johnson A, Lamagni T, Chronias A, Sheridan E: Trends among pathogens reported as causing bacteraemia in England, 2004-2008. Clin Microbiol Infect 2011, 17:451-458. doi:10.1111/j.1469-0691.2010.03262.x.

12. Edgeworth JD: Has decolonization played a central role in the decline in UK methicillin-resistant Staphylococcus aureus transmission? A focus on evidence from intensive care. J Antimicrob Chemother 2011, 66(suppl 2):41-47. doi:10.1093/jac/dkq325.

13. Jarlier V, Trystram D, Brun-Buisson C, Fournier S, Carbonne A, Marty L, Andremont A, Arlet G, Buu-Hoi A, Carlet J, Decré D, Gottot S, Gutmann L, Joly-Guillou ML, Legrand P, Nicolas-Chanoine MH, Soussy CJ, Wolf M, Lucet JC, Aggoune M, Brücker G, Régnier B: Curbing methicillin-resistant staphylococcus aureus in 38 French hospitals through a 15-year institutional control program. Arch Intern Med 2010, 170:552-559. doi:10.1001/archinternmed.2010.32. 
14. Smith A, Christie P, Stari T, Kavanagh K, Robertson C, Reilly J, Gould I, MacKenzie F, Masterton R: The value of nasal swabbing versus full body screening or clinical risk assessment to detect MRSA colonisation at admission to hospital (NHS Scotland MRSA screening pathfinder programme: final report). Glasgow: Health Protection Scotland; 2011

15. Ritchie K, Bradbury I, Eastgate J, Foster L, labal K, MacPherson K, McCarthy T, Mclntosh H, Nic Lochlainn E, Reid M, Taylor J: The clinical and cost effectiveness of screening for meticillin-resistant Staphylococcus aureus (health technology assessment report 9). Glasgow: Quality Improvement Scotland; 2007.

16. Collins J, Raza M, Ford M, Hall L, Brydon S, Gould FK: Review of a threeyear meticillin-resistant Staphylococcus aureus screening programme. J Hosp Infect 2011, 78:81-85.

17. Parienti JJ, Cattoir V, Thibon P, Lebouvier G, Verdon R, Daubin C, du Cheyron D, Leclerca R, Charbonneau P: Hospital-wide modification of fluoroquinolone policy and meticillin-resistant Staphylococcus aureus rates: a 10-year interrupted time-series analysis. J Hosp Infect 2011, 78:118-122

18. Wagner AK, Soumerai SB, Zhang F, Ross-Degnan D: Segmented regression analysis of interrupted time series studies in medication use research. J Clin Pharm Ther 2002, 27:299-309.

19. Grimshaw J, Campbell M, Eccles M, Steen N: Experimental and quasiexperimental designs for evaluating guideline implementation strategies. Fam Pract 2000, 17(Suppl 1):11-16.

20. Stone SP, Cooper BS, Kibbler CC, Cookson BD, Roberts JA, Medley GF, Duckworth G, Lai R, Ebrahim S, Brown EM, Wiffen PJ, Davey PG: The ORION statement: guidelines for transparent reporting of outbreak reports and intervention studies of nosocomial infection. J Antimicrob Chemother 2007, 59:833-840. doi:10.1093/jac/dkm055.

21. Kallen AJ, Mu Y, Bulens S, Reingold A, Petit S, Gershman K, Ray SM, Harrison LH, Lynfield R, Dumyati G, Townes JM, Schaffner W, Patel PR, Fridkin SK: Health care-associated invasive MRSA infections, 2005-2008. JAMA 2010, 304:641-647. doi:10.1001/jama.2010.1115.

22. Murchan S, Auken HM, O'Neill G, Ganner M, Cookson BD: Emergence, spread and characterisation of phage variants of epidemic meticillinresistant staphylococcus aureus-16 (EMRSA-16) in England and Wales. J Clin Microbiol 2004, 57:345-346.

23. Perencevich EN: Decline in invasive MRSA InfectionWhere to Go from here? JAMA 2010, 304(6):687-689. doi:10.1001/jama.2010.1125.

doi:10.1186/2047-2994-2-2

Cite this article as: Sarma et al:: Impact of universal screening on MRSA bacteremias in a single acute NHS organisation (2006-12): interrupted time-series analysis. Antimicrobial Resistance and Infection Control 2013 2:2.

\section{Submit your next manuscript to BioMed Central and take full advantage of:}

- Convenient online submission

- Thorough peer review

- No space constraints or color figure charges

- Immediate publication on acceptance

- Inclusion in PubMed, CAS, Scopus and Google Scholar

- Research which is freely available for redistribution 